Rev. Elev. Méd. Pays trop., 1979, 32 (2) : 149-153

\title{
Les coccidies bovines en République Centrafricaine
}

\author{
par J. VERCRUYSSE $(*)$
}

\begin{abstract}
RÉSUMÉ
Dans la partie occidentale de R. C. A., 1700 veaux et 400 zébus adultes ont été examinés pour établir la prévalence de la coccidiose. Chez les veaux, on trouve une infestation moyenne sur toute l'année de 37 p. 100 et chez les adultes de 15 p. 100 . Les différentes espèces rencontrées ont été déterminées (II) et leur importance relative a été estimée.
\end{abstract}

\section{INTRODUCTION}

Dans la partie occidentale de la République Centrafricaine (R. C. A.) existent environ 700000 bovins appartenant à l'espèce zébu (races Mbororo, Foulbé, Goudalé). On y rencontre également quelques milliers de taurins (races Ndama et Baoulé), mais ils ne sont pas compris dans cette étude.

Les zébus sont élevés en élevage extensif par les Peuls Mbororo : ceux-ci pratiquent la transhumance pendant la saison sèche qui dure quatre mois et ils établissent un campement fixe pendant la saison des pluies qui dure environ huit mois, soit de mars à novembre. La végéta- tion dans la zone considérée est du type soudanoguinéen.

La mortalité chez les veaux est élevée et atteint 35-40 p. 100. Puisque l'étiologie n'en est pas connue, on a recherché quel rôle la coccidiose pourrait avoir dans le taux de mortalité ou dans la pathologie intestinale.

Pendant 11 mois, 1700 veaux et 400 zébus adultes ont été examinés, pour la recherche des nématodes, des trématodes et des coccidies. Le présent travail expose le résultat de cette recherche en ce qui concerne les coccidies.

(*) Kempische Veldweg 94, 2230 Schilde, Belgique. Expert-associé F. A. O., Bouar, R. C. A.

TABLEAU I-La prévalence de l'ınfectıon coccidienne chez les veaux en E.C.A. (p.100)

\begin{tabular}{|c|c|c|c|c|c|c|c|c|c|c|c|c|}
\hline $\begin{array}{l}\text { Age des } \\
\text { veaux } \\
\text { (mois) }\end{array}$ & $\begin{array}{l}\text { Oct. } \\
77\end{array}$ & Nos. & Déc. & $\begin{array}{r}\mathrm{Jan} \\
78 \\
\end{array}$ & Fév. & Mars & $A V I$. & $\mathrm{Ma}_{1}$ & Juin & JuIl. & Août & $\left\{\begin{array}{c}\text { Aoyeint } \\
\text { sur } \\
11 \text { mons }\end{array}\right.$ \\
\hline$<1$ & 60 & 27 & 13 & 6 & 7 & $-*$ & 7 & 24 & 30 & 61 & 23 & 25 \\
\hline $1-3$ & 64 & 40 & 19 & 38 & 12 & 28 & 16 & 29 & 39 & 70 & 64 & 42 \\
\hline $3-6$ & 54 & 25 & 75 & 27 & 20 & 28 & $2+4$ & 22 & 42 & 52 & 43 & 38 \\
\hline $6-12$ & 33 & 69 & $-*$ & 36 & $2 ?$ & $-*$ & $-*$ & 48 & 31 & 56 & 51 & 38 \\
\hline
\end{tabular}




\section{MATÉRIEL ET MÊTHODE}

Dans un rayon de $250 \mathrm{~km}$ autour de Bouar, une centaine de troupeaux ont été visités et en moyenne, dans chaque campement, 20 échantillons ont été prélevés par voie rectale. Les échantillons furent conservés soit à une température de $4^{\circ} \mathrm{C}$ soit dans le formol à 5 p. 100 .

La méthode de flottaison, à base d'une solution saturée de sel, fut utilisée pour la recherche des oocystes.

Les mensurations au micromètre et l'étude du temps de la sporulation des oocystes ont été faites selon la méthode de CHRISTENSEN (4). Après flottaison dans la saccharose à 40 p. 100 , les oocystes de la surface furent prélevés avec une anse de platine. Ensuite ils furent placés dans une goutte pendante sur une lamelle carrée (22 $\mathrm{mm}$ de côté) inversée sur une lame à concavité. Par flottaison secondaire, les oocystes adhèrent à la lamelle. On évite ainsi toute distorsion par pression, et les oocystes s'orientent avec leur grand axe parallèle à la surface inférieure de la lamelle, ce qui facilite les mensurations. Pendant la sporulation ( 1 à 6 jours) on a maintenu une température de 25 à $27^{\circ} \mathrm{C}$.

L'identification des oocystes a été basée sur la clé utilisée par PELLERDY (8) utilisant comme caractéristiques de différenciation la forme, la couleur, les dimensions et le temps de sporulation des oocystes.

\section{RÉSULTATS}

a) Prévalence de la coccidiose chez les veaux

Au total 1700 veaux ont été examinés pour les coccidies entre octobre 1977 et août 1978 (en moyenne 160 par mois).

Il y a une fluctuation selon les saisons. Entre décembre et mai, le taux d'infestation reste inférieur à 30 p. 100 (saison sèche) tandis que le mois de juillet connaît le taux le plus élevé (62 p. 100). Sur toute l'année, l'infestation moyenne est 37 p. 100.

Ce sont les veaux de 1 à 3 mois qui semblent les plus fortement infestés. Le nombre d'oocystes est faible (OPG $<1000$ ) et il est rare de trouver des veaux avec une forte infestation.

b) Prévalence de la coccidiose chez les bouvillons, génisses et adultes

Il semble qu'elle ait beaucoup moins d'importance que chez les veaux. En moyenne on trouve 15 p. 100 des bêtes positives.

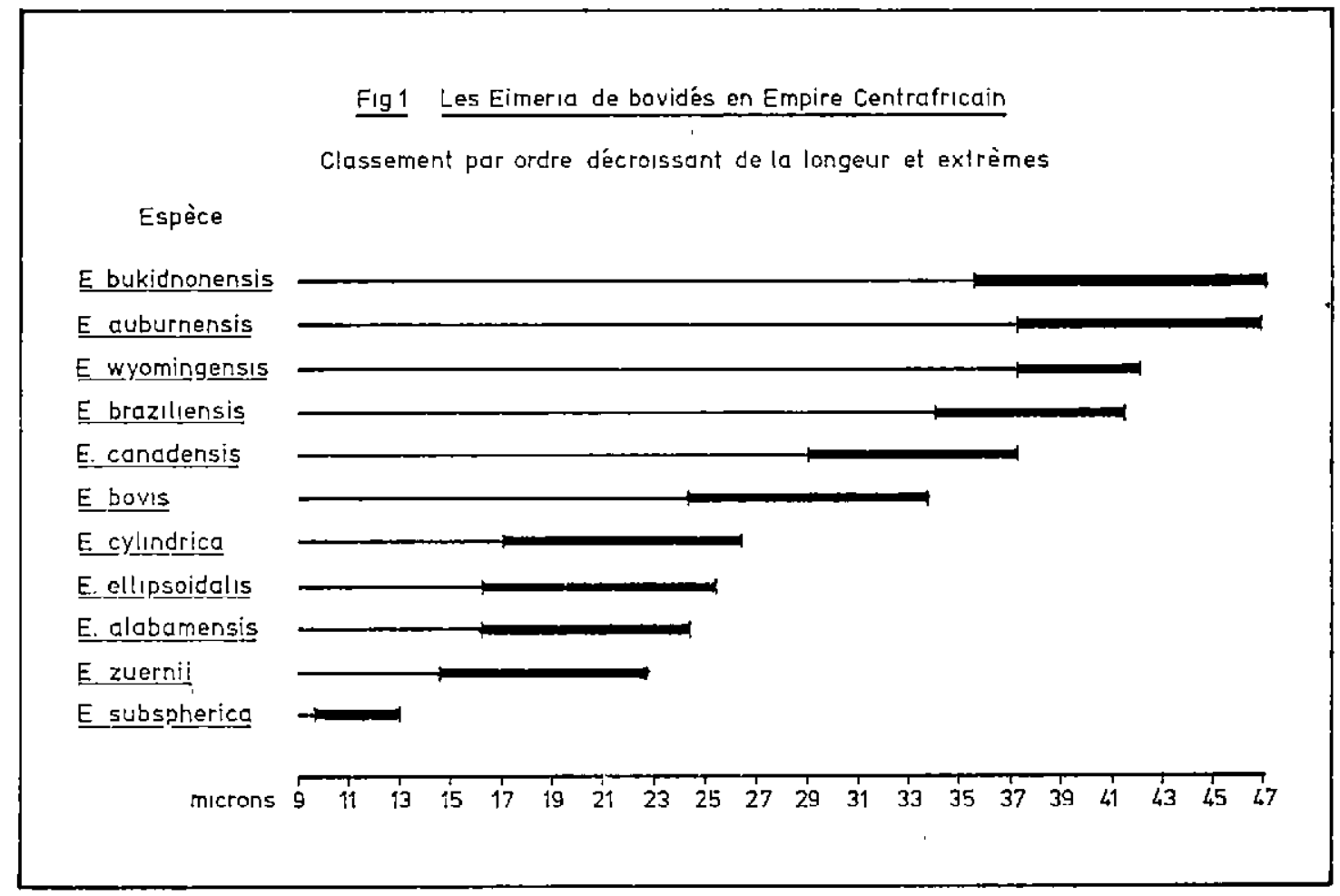


ThbLEAU II-Caractéristiques morphologiques et biologiques des coccidies bovines en E.C.A.

\begin{tabular}{|c|c|c|c|c|c|c|c|c|c|c|}
\hline Eupree & $\begin{array}{l}\text { Nombre } \\
\text { doochere } \\
\text { menure }\end{array}$ & $\begin{array}{l}\text { Longuevz } \\
\text { moyenne }\end{array}$ & Amplutude & $\begin{array}{l}\text { Ecart } \\
\text { sype }\end{array}$ & $\begin{array}{l}\text { Largeur } \\
\text { mayenne }\end{array}$ & Amplitude & $\begin{array}{l}\text { Ecast } \\
\text { type }\end{array}$ & $\begin{array}{l}\text { Parametre } \\
\text { de Iorme: } \\
\text { moyenne:- }\end{array}$ & Amplitude & $\begin{array}{c}\begin{array}{c}\text { Temps de } \\
\text { eporufation, } \\
\text { heurea }\end{array} \\
\end{array}$ \\
\hline E. alabamennis & 110 & $202 \pm 0219$ & $162-24.3$ & 2,30 & $14,3 \pm 0,054$ & $113-178$ & 057 & 071 & $060-0 \mathrm{B4}$ & $96-120$ \\
\hline E. auburnendel & 330 & $10.7 \pm 0.178$ & $37.3=46.9$ & 323 & $257 \pm 0.063$ & $21.0-29.7$ & J. 15 & 0.62 & $051-0.72$ & $48-72$ \\
\hline E. bovila & 330 & $255 \pm 0076$ & $24.3-33.7$ & 1.38 & $20.2 \pm 0.070$ & $17,2-243$ & 127 & 0.70 & $0.57-0.88$ & 4. -72 \\
\hline E. brazillensia & 50 & $37.2 \pm 0.337$ & $340-40.5$ & 238 & $26.0 \pm 0.133$ & $24,3-292$ & 093 & 0.74 & $0.66-0.81$ & -- \\
\hline E. bukidnonensis & 60 & $423 \pm 0.287$ & $35.6-47.0$ & 2,21 & $31.9 \pm 0 \quad 179$ & $29.2-35.6$ & 1.38 & 0.75 & $0.70 \cdot 0.91$ & $\cdots$ \\
\hline E canadensis & 280 & $33.2 \pm 0.115$ & $29.1-37.3$ & 1.92 & $24 . i \pm 0.013$ & $19.1 \cdot 25.9$ & 0.22 & 071 & $061-0.84$ & $72-120$ \\
\hline E. cylindrica & 20 & $22.7 \pm 0.396$ & $170-26: 4$ & 157 & $135 \pm 0546$ & $113-14.6$ & 2.50 & 0.59 & $0.52-0.76$ & $-\bullet$ \\
\hline E. ellipeordatle & 310 & $216 \pm 0.100$ & $162-25,4$ & 177 & $155 \pm 0075$ & $113-17 B$ & 132 & 070 & $0.60-090$ & $48 \cdot 72$ \\
\hline E. aubspherica & 65 & $11.4 \pm 0.105$ & $97-13.0$ & 085 & $108 \pm 208 ?$ & $9.0-21.3$ & 0.66 & 0.94 & $085-1$ & $\cdots$ \\
\hline E, wyomingensis & 32 & $395 \pm 0.250$ & $37,3-521$ & 140 & $28.0 \pm 0125$ & $27.4-30.8$ & 0.71 & 0.71 & $0.68-0.71$ & $-\cdot$ \\
\hline E. zuernin & 320 & $100 \pm 0.083$ & $14,6-22,7$ & 1,50 & $161 \pm 0 \quad 092$ & $130-194$ & 1.65 & 0.89 & $0.73 \cdot 1$ & $48-72$ \\
\hline
\end{tabular}

TABLEAJ III-Clë d'identification des coccidles bovines en E.C.A.:

\begin{tabular}{|c|c|c|c|c|c|c|}
\hline Enpic* & Longueur $(\mu)$ & Forma & $\begin{array}{l}\text { Tempo de } \\
\text { "porulation } \\
\text { (heures) }\end{array}$ & Micropyle & Couleur & $\begin{array}{c}\text { Caractériatuques } \\
\text { opteniliquez }\end{array}$ \\
\hline E., Ubbepherica & $<15$ & -ub-Iphérique & $120-144$ & zbeent & incolore & néant \\
\hline E. slabemonslo & \multirow{4}{*}{15225} & pyriforme & $96-120$ & Sbeent & incolore & grande varlation do forme \\
\hline E, ellipsoidalla & & ellipioldel & $19 \cdot 72$ & abeent & incolore & néant: \\
\hline E. cylindslea & & cylindxlq̨uo & $49-72$ & abgent & incolore & néant \\
\hline E. Euernll & & ab+ephérique & $48=72$ & Eboent & Incolore & 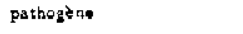 \\
\hline E bovis & \multirow{2}{*}{$25 \div 35$} & avilorme & $4 a-72$ & płésent & $\begin{array}{l}\text { psle, jaunatra. } \\
\text { verdalre - brun }\end{array}$ & pethogene \\
\hline E. canadenala & & ellapsoidal & $72 \cdot 120$ & présent & Jaunatse - brun & néant \\
\hline E. auburnentia & \multirow{4}{*}{$>35$} & ovifornie & $19-72$ & pretent & $\begin{array}{l}\text { jainative-brun } \\
\text { pale ou foncé }\end{array}$ & oocyote allange \\
\hline E. wyomlagasolo & & avilarme & $72-120$ & prtsenl & $\begin{array}{l}\text { Jaunatre et } \\
\text { verdatze -brun }\end{array}$ & oocyete trapu \\
\hline E. brazilieneld & & ellpsoidal & $144-168$ & préaent & jaunatre - brun & capmule polatre \\
\hline E. bukıd nonent 25 & & pyrtiorme & $120-168$ & prósen' & brus & $\begin{array}{l}\text { Parol fpasse } \\
\text { strute radialemert: }\end{array}$ \\
\hline
\end{tabular}

*D'aproa lea travaux at Christenuen, Lee \& Pellerdy

\section{c) Espèces rencontrées}

L'identification des différentes coccidies n'a pas posé de grandes difficultés.

Onze différentes espèces d'Eimeria ont été identifiées, ce sont : $E$. alabamensis, $E$. auburnensis, E. bovis, E. braziliensis, E. bukidnonensis, E. canadensis, E. cylindrica, E. ellipsoidalis, $E$. subspherica, E. wyomingensis, E. zuernii.

La présence d'E. braziliensis, E. cylindrica, $E$. subspherica et $E$. wyomingensis est rare : il ne nous a pas été possible de mesurer beaucoup d'oocystes ni d'établir le temps de sporulation. E. bukidnonensis a été identifiée seulement dans un troupeau chez deux veaux. Malheureusement les selles étaient conservées dans le formol : il fut donc impossible de faire le test de sporulation. Au contraire, E. alabamensis, E. auburnensis,
E. bovis, E. canadensis, E. ellipsoidalis et E. zuernii sont très fréquentes en $\mathbf{R}$. C. A., et on a pu mesurer plusieurs centaines d'oocystes de chaque espèce et en établir le temps de sporulation.

On a mesuré au total 1910 oocystes et on a comparé les résultats avec ceux de LEE (7), CHRISTENSEN (4) et éventuellement avec ceux d'autres publications originales.

La description et les dimensions des coccidies examinées correspondent aux études faites antérieurement par YAKIMOFF (10), CHRISTENSEN (4) et $\operatorname{LEE}(6,7)$. En R. C. A., l'infection coccidienne des bovins se caractérise par une grande diversité étiologique. Il n'est pas rare de trouver des veaux infestés par 4 à 5 différentes espèces d'Eimeria: l'association d'E. auburnensis, E. bovis, E. canadensis, E. ellipsoidalis et E. zuer- 
nii est très courante. La coccidie la plus fréquemment rencontrée était E. ellipsoidalis.

\section{DISCUSSION}

En République Centrafricaine, la coccidiose chez les veaux et à un moindre degré chez les bovins adultes est loin d'être négligeable. Ses caractéristiques sont: fluctuation selon les saisons; répartition inégale suivant les troupeaux ; l'évolution clinique fréquemment asymptomatique (parfois on trouve des troupeaux infestés à 100 p. 100 ne présentant aucun signe clinique) et la grande diversité d'espèces. CABARET (3), en Mauritanie, constate une fluctuation selon les saisons chez les veaux avec une pointe de 72 p. 100 entre octobre et décembre. BOUCHET (1) trouve dans l'Ouest Centrafricain sur 984 veaux 14 p. 100 d'infection coccidienne sur l'ensemble de l'année. Cette infection semble surtout prendre de l'ampleur à l'âge de 3 mois (27,4 p. 100).

Les Eimeria provoquant des symptômes cliniques sont $E$. bovis et $E$. zuernii. Cependant on a trouvé quatre veaux avec diarrhée sévère mais non sanguinolente infestés par E. auburnensis. Nous n'avons pas pu déterminer si celle-ci était seule en cause ou si c'était plutôt son association avec Strongyloides papillosus. On n'a jamais observé de signes cliniques nerveux tels que JULIAN (5) les a décrits.

Le tableau III montre qu'en se basant sur la longueur et la forme on peut faire une identification des oocystes bovins. Cependant certains oocystes d'E. alabamensis qui est connue pour sa grande variation de forme, peuvent être confondus avec ceux d'E. ellipsoidalis ou E. cylindrica mais chez la première la période de sporulation dépasse les $96 \mathrm{~h}$. Certaines formes d'E. ellipsoidalis qu'on trouve en grand nombre chez des veaux en bonne santé ressemblent à celles d'E. zuernii qui est souvent associée avec une coccidiose clinique. La forme ovoïdale plus allongée et le temps de sporulation plus court permettent de différencier $E$. auburnensis d' $E$. wyomingensis. La principale caractéristique morphologique d'E. braziliensis est sa capsule polaire. Chez E. bukidnonensis on observe toujours une paroi épaisse striée radialement.

ll serait intéressant d'étudier les oocystes de coccidies des grands herbivores sauvages pour essayer de trouver l'origine des différentes espèces trouvées chez les zébus en R.C. A. Sont-elles indigènes ou ont-elles été importées ?

Il semble que la coccidiose en R.C. A., malgré la prévalence élevée de l'infection, n'est pas une des causes principales de mortalité des veaux sauf lorsqu'elles accompagnent d'autres parasitoses.

\section{REMERCIEMENTS}

L'auteur tient à remercier vivement le $D^{c}$ LINDLEY, Directeur du Projet Santé Animale (F.A.O.) et le $\mathrm{D}^{\mathrm{r}}$ THIENPONT, Janssen Pharmaceutica, pour les conseils scientifiques qu'ils ont bien voulu lui donner.

\section{SUMMARY}

\section{Bovine coccidiosis in Centrafrican Republic}

During 11 months, 1700 calves and 400 adult zebu cattle were examined in west Centrafrican Republic.

Coccidiosis occurs in 37 p. 100 of the former and in 15 p. 100 of the latter.

Eleven species were identified and their relative importance is discussed.

\section{RESUMEN}

\section{Los coccidios bovinos en la República Centroafricana}

En la parte occidental de la República Centroafricana, se examinaron 1700 terneros y 400 cebues adultos para dernostrar el predominio de la coceidiosis. En los terneros, se encuentra una infestación media durante todo el año de 37 p. 100 y en los adultos de 15 p. 100 . Se determinaron las diferentes especies encontradas (11) y se estimó su importancia relativa. 


\section{BIBLIOGRAPHIE}

1. BOUCHET (A.). Communication personnelle, 1968.

2. BRUCE (E. A.). Bovine coccidiosis in British Columbia with a description of the parasite, Eimeria canadensis sp. n. J. am. vet. med. Ass., 1921, 58 : 638-662.

3. CABARET (J.). Note sur le parasitisme dû aux nématodes et aux coccidies chez les espèces domestiques dans la région de Kaedi (Mauritanie). Rev. Elev. Méd. vét. Pays trop., 1976, 29 : 221-226.

4. CHRISTENSEN (J. F.). The oocysts of coccidia from domestic cattle in Alabama (U.S. A.) with descriptions of two new species. J. Parasit., 1941, 27 : 203-220.

5. JULIAN (R. J.) et HARRISON (K. B.). Nervous signs in bovine coccidiosis. Modern vet. Pract., 1976, 57 : 711-718,
6. LEE (R. P.). The occurrence of the coccidian Eimeria bukidnonensis, Tubangui 1931, in Nigerian cattle. $J$. Parasit., 1954, 40 : 464-466.

7. LEE (R. P.) et ARMOUR (J.). The coccidia oocysts of Nigerian cattle. Brit. vet. J., 1959, 115 : 1-12.

8. PELLÉRDY (L.). Coccidia and coccidioses. Budapest, Akadémiai kladé, 1965,

9. TUBANGUI (M. A.). Eimeria bukidnonensis a new coccidium from cattle and other coccidial parasites of domesticated animals. Philipp J. Sci., 1931, 44 : 253271.

10. YAKIMOFF (W. L.). La coccidiose des animaux domestiques dans' l'Azerbaidjan (Transcaucasia). Annls Soc. Belge Méd. trop., 1933, 13 : 93-130. 